\title{
Light-particle single ionization of argon: Influence of the projectile charge sign
}

\author{
S. Otranto ${ }^{1, *}$ and R. E. Olson ${ }^{2}$ \\ ${ }^{1}$ CONICET and Departamento de Física, Universidad Nacional del Sur, 8000 Bahía Blanca, Argentina \\ ${ }^{2}$ Department of Physics, Missouri University of Science and Technology, Rolla, Missouri 65409, USA
}

(Received 11 May 2009; published 28 July 2009)

\begin{abstract}
The ionization of the $3 p$ orbital of argon by incident electrons and positrons is studied by means of the post version of the continuum distorted wave-eikonal initial-state model. Results are presented at both 200 and 500 $\mathrm{eV}$ impact energies for conditions amenable to present experiments. Differences in the fully differential cross sections (FDCSs) are analyzed and the influence of the projectile charge sign on the emission dynamics is discussed. The FDCSs are found to display the classic binary plus recoil peak structure at $500 \mathrm{eV}$, but transition to a more complicated four-lobed structure at the lower impact energy.
\end{abstract}

DOI: 10.1103/PhysRevA.80.012714

PACS number(s): 34.80.Dp, 34.10.+x

\section{INTRODUCTION}

Although fully differential data for $(e, 2 e)$ reactions were published 4 decades ago in the pioneering work of Ehrhardt et al. [1], it has been only a few years since similar data have become available for positron impact. This time gap can be related to the low positron beam intensities available in atomic physics laboratories, making experimental studies extremely difficult. The first effort to get fully differential data for positron-argon collisions was performed by Köver et al. and dates back to 1993 [2]. In successive years, these authors also obtained and presented triply differential cross sections for positrons scattered near zero degrees for the $\mathrm{H}_{2}$ target $[3,4]$. More recently, the experimental group of DuBois succeeded in obtaining fully differential cross-section (FDCS) angular distributions for positron-argon ionizing collisions at $500 \mathrm{eV}$, with positrons scattered to nonzero scattering angles $[5,6]$.

From the theoretical side, FDCSs for electron and positron impact ionizations of atomic hydrogen were presented by Brauner and Briggs in 1993 [7] using the Born3C model. Afterwards, the data measured by Köver et al. in Ref. [2] were analyzed by Sparrow and Olson using the classical trajectory Monte Carlo method [8]. Later, Fiol et al. [9] applied a Born3C-type model similar to that of Brauner and Briggs in order to compare to the data of Ref. [2].

The overall dynamics of matter-antimatter collisions were reviewed by Schultz et al. [10]. In general, the differences in the total and single differential cross sections showed relatively smooth behavior in energy or angle [11]. However, the targets investigated were simple ones such as hydrogen and helium that possessed isotropic electronic distributions. From the early work that includes the Brauner and Briggs calculations, one is lead to expect that the FDCS for positron impact should possess the "classic" two-lobe binary plus recoil peak structure. Moreover, the comparison of FDCS between $e^{+}$ and $e^{-}$should be relatively straightforward with an enhanced binary peak magnitude for positron impact relative to that for electrons because of the post-collision interaction between projectile and ionized electron. Likewise, a larger recoil peak for electrons is expected because of the repulsive electronelectron interaction. We show in this work that such extrapolations are oversimplified for a target such as argon that has a nonisotropic outer shell electronic structure.

In the following, we report theoretical FDCS for electron and positron impact single ionizations of argon at impact energies of 200 and $500 \mathrm{eV}$ for different emission energies and projectile scattering angles. The energies and projectile scattering angles were chosen to be compatible with experimental observation $[5,6]$. One goal of this work is to illustrate the differences due to projectile charge in the FDCS, remembering that at the first Born-approximation level, all cross sections are identical.

In the next section, we briefly describe the theoretical method used. Results are shown in Sec. III and finally conclusions are drawn in Sec. IV. Atomic units are used throughout this work unless otherwise stated.

\section{THEORETICAL METHOD}

We have employed the continuum distorted wave-eikonal initial-state (CDW-EIS) model, which has been developed and extensively used in ion-atom scattering for more than 2 decades [12-17]. Over the years, this model has been extended to study electron-impact ionization of atomic hydrogen [18] and, very recently, to single ionization of the $3 p$ orbital of argon [19]. The FDCS in the CDW-EIS model is given by

$$
\frac{d^{5} \sigma}{d E_{2} d \Omega_{2} d \Omega_{1}}=N_{e e}(2 \pi)^{4} \frac{k_{1} k_{2}}{k_{0}} \sum_{3 p_{0}, 3 p_{1}, 3 p_{-1}}\left|T_{f i}\right|^{2} .
$$

Here, $N_{e e}$ represents the number of identical electrons in the shell, $k_{1(2)}$ represents the momentum of the receding projectile and emitted electron, respectively, and $k_{0}$ represents the impinging projectile momentum. The exact transition amplitude in the Distorted wave formalism $\left(T_{f i}\right)$ [20] in Eq. (1) is represented by

$$
\begin{gathered}
T_{f i}=T_{f i}^{I}+T_{f i}^{I I}, \\
T_{f i}^{I}=\left\langle\chi_{k_{1}}^{-} \chi_{k_{2}}^{-} D\left(r_{12}\right)\left|1 / r_{12}-V\left(r_{1}\right)\right| \Phi_{i} \varphi_{i}\right\rangle,
\end{gathered}
$$

\footnotetext{
*sotranto@uns.edu.ar
} 


$$
T_{f i}^{I I}=\left\langle{\chi \bar{k}_{1}}_{\overline{k_{2}}}^{-} D\left(r_{12}\right)\left|\nabla_{1} \nabla_{12}-\nabla_{2} \nabla_{12}\right|\left(\varepsilon_{1} \varepsilon_{12}-1\right) \Phi_{i} \varphi_{i}\right\rangle .
$$

The first amplitude is just the Born initial state-3 distorted waves final state (Born-3DW) approximation while the second amplitude contains all the higher-order corrections to this approximation. The wave functions $\overline{\chi_{k_{1,2}}}$ are distorted waves which are generated from central potentials for the ion core. To represent the interaction of the electrons with the remaining ionic core, we consider the usual static screening potentials plus a local approximation for the electronic exchange. In this case, we have chosen the form provided by Gianturco and Scialla [21] which is particularly suited for particles in the low-energy regime

$$
\begin{aligned}
V_{E}= & 0.5\left[E-V_{s t}(r)+\frac{3}{10}\left[3 \pi^{2} \rho(r)\right]^{2 / 3}-\left\{\left(E-V_{s t}(r)\right.\right.\right. \\
& \left.\left.\left.+\frac{3}{10}\left[3 \pi^{2} \rho(r)\right]^{2 / 3}\right)^{2}+4 \pi \rho(r)\right\}^{1 / 2}\right] .
\end{aligned}
$$

Here, $V_{s t}(r)$ is the static screening potential which is calculated with ionic orbitals and $\rho(r)$ is the electron charge density.

For the positron-core interaction, we only use the static screening potential. The infinite partial waves are considered in $\chi_{k_{1,2}}^{-}$, since the Kummer functions associated to the corresponding asymptotic charges are corrected in the required number of partial waves until convergence to the Coulomb case is achieved. For $\Phi_{I}$, we have chosen a Clementi-Roetti wave function [22] and $V$ represents the core potential seen by the impinging electron or positron. The latter is the only static screening potential calculated with the atomic orbitals for positron impact while a Gianturco-Schialla exchange term is also added for electron impact. The distortion $D\left(r_{12}\right)$ is given by a Kummer function as shown in Ref. [19]. In Eq. (2), $\varphi_{I}$ represents the incident plane wave for the projectile while $\varepsilon_{j}$ are the eikonal distortions.

The calculation of the transition amplitude has been developed by direct six-dimensional (6D) numerical integration over the coordinates using the "VEGAS" adaptive Monte Carlo scheme together with the wave-packet approach developed by Malcherek and Briggs [23]. This numerical scheme was also implemented in recent years by Götz et al. [24] in $(e, 3 e)$ studies. The implementation of the method and the numerical accuracy were tested by using the hydrogen target (see Ref. [19]). Here, the Monte Carlo integration was successfully tested against a previously developed code based on Nordsieck integrals and a three-dimensional 3D numerical integration scheme. Since we are concerned with very asymmetric collisions, for electron impact, we only include the direct amplitude neglecting the possibility of exchange between the impinging projectile and the active electron.

\section{RESULTS}

First, we compare our CDW-EIS results for $500 \mathrm{eV}$ electron-impact ionization of Ar to the recent theoretical and experimental data by Kheifets et al. [25]. Those authors employed a distorted-wave Born approximation together with a Gamow factor (DWBA-G) to account for the post-collision

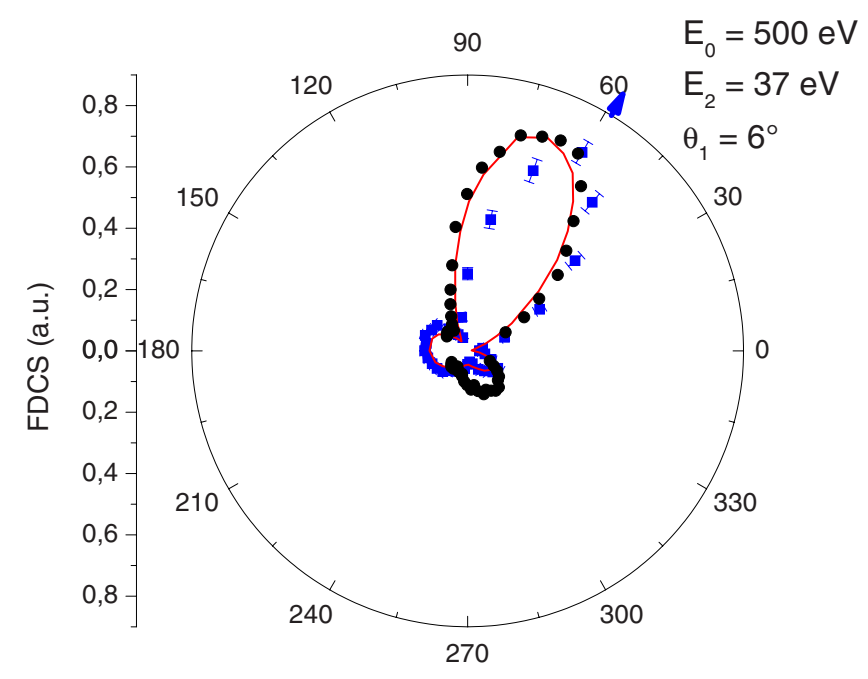

FIG. 1. (Color online) FDCS for $500 \mathrm{eV}$ electron-impact ionization of argon. The emission energy considered is $37 \mathrm{eV}$ and the projectile scattering angle is $6^{\circ}$ clockwise. The experimental data from Kheifets et al. (Ref. [25]) are shown with dots normalized to the CDW-EIS theory (squares with statistical error bars). The DWBA-G results of Ref. [25] have been included for comparison and scaled by a factor of 1.15 and are given by the solid line. Small arrow indicates the momentum-transfer direction.

interaction (PCI) among the receding electrons. At this impact energy and for a wide range of momentum transfers, they obtained good agreement with the experimental data for the noble gases $\mathrm{He}, \mathrm{Ne}$, and Ar. In Fig. 1, we consider the emitted electron energy of $37 \mathrm{eV}$ while the projectile scattering angle is $6^{\circ}$. We have chosen this configuration since it corresponds to the smallest momentum transfer studied in Ref. [25] for the collision system under study. We observe that the CDW-EIS theory provides results in good agreement with the DWBA-G. The DWBA-G results have been multiplied by a scaling factor of 1.15 to normalize them to our CDW-EIS results at the binary peak. Furthermore, we notice that for both theories, as well as for the experimental data, the binary peak is located very close to the direction along which the momentum-transfer vector is defined. However, it should be pointed out that neither DWBA-G nor CDW-EIS provides an accurate description of the data at larger angles. From the experimental and theoretical data of Ref. [25], we infer that this trend is still valid for other emission energies which correspond to larger values of the momentum transfer.

One thing we noticed during the calculation of the present figure was that as the cross-section magnitude decreases, the number of evaluations required to achieve convergence grows drastically. The CDW-EIS calculation shown in Fig. 1 required $250 \times 10^{6}$ evaluations of the integrand to achieve an estimated error of about $6 \%$ at the top of the binary peak as can be inferred from the associated error bars. The geometric asymmetric configurations that will be shown below for less energetic emitted electrons were obtained with an estimated error of less than $5 \%$ by using $140 \times 10^{6}$ evaluation points.

In Fig. 2, we present FDCS for $500 \mathrm{eV}$ positron and electron impact with the incident projectile scattered to $3^{\circ}$ clockwise. The ionized electron energies are 2.4, 7.3, and $20 \mathrm{eV}$. The experimental positron data for 2.4 and $7.3 \mathrm{eV}$ ionized 

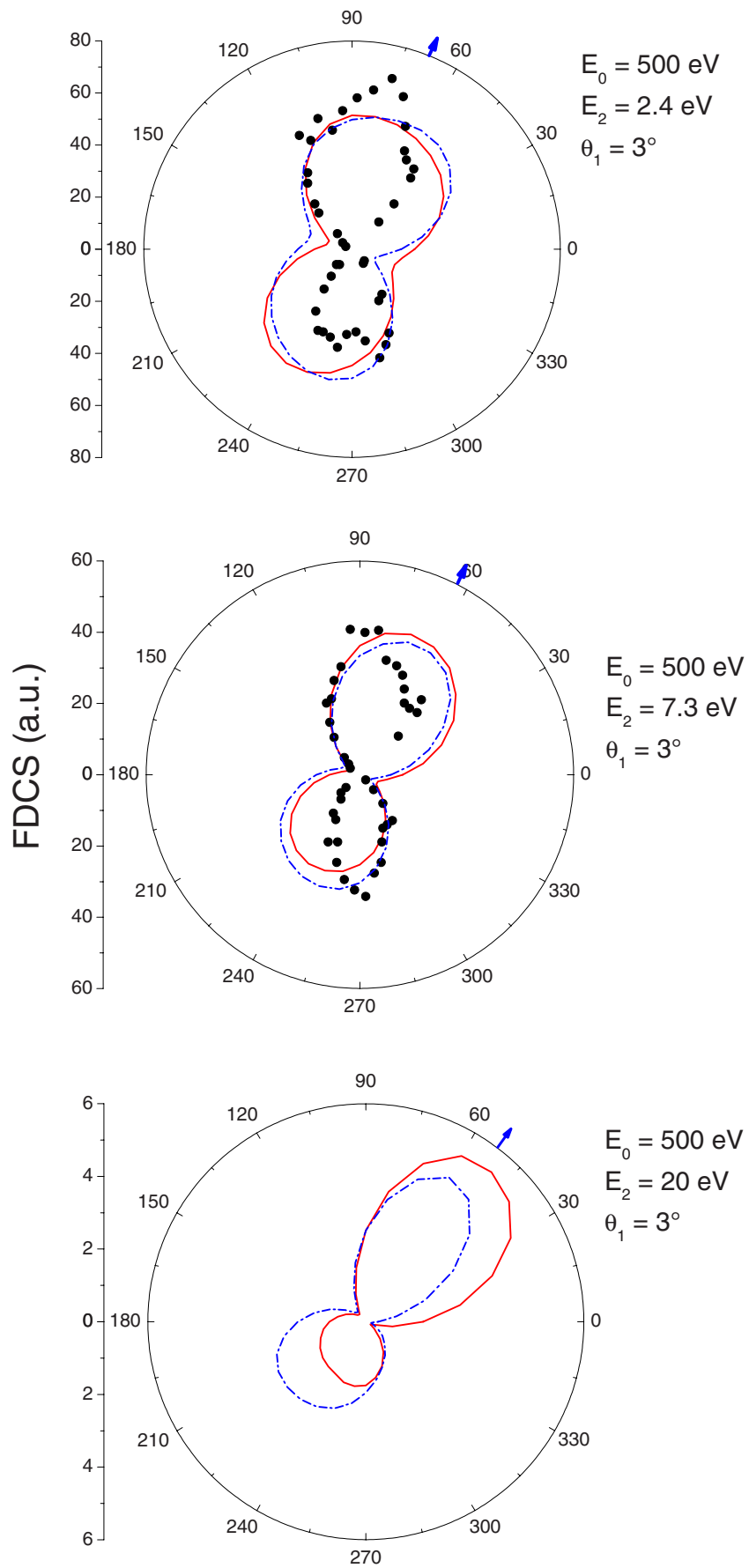

FIG. 2. (Color online) FDCSs for $500 \mathrm{eV}$ positron (solid line) and electron (dot-dashed line) impact ionizations of argon. The emission energies considered are 2.4, 7.3, and $20 \mathrm{eV}$ and the projectile scattering angle is $3^{\circ}$ clockwise. The experimental data are that of de Lucio et al. [5]. Small arrows indicate the momentumtransfer direction

electrons are from de Lucio et al. [5]. Our calculations have not been convoluted for experimental acceptance angles and energies. In accordance with the results showed in Fig. 1, our positron and electron calculations display a binary peak position very close to that of the direction of momentum transfer. At first glance, the agreement between experiment and theory is poor with the calculations displaying much broader binary and recoil peaks than the data. However, it must be remembered that the position-sensitive channel-plate detector used in the measurements is only sensitive to electrons emitted in the angular range $45^{\circ}<\theta_{e}<135^{\circ}$ in the absence of any extraction field. According to the authors, the extraction field allows low-energy electrons ejected outside this angular range to be detected. However, since the extraction field affects all the emitted electrons, the reported geometric angles can be quite different from the "true" electron emission angles [5] and should result in an angular profile that is preferentially focused toward $90^{\circ}$. It is not possible to deconvolute the data to obtain the true electron emission angles due to the large angular and energy acceptances of the detector. Note that in the 2.4 and $7.3 \mathrm{eV}$ data, the centroid energies of detections were -4.4 and $6.4 \mathrm{eV}$, respectively.

Another quantity observed was the energy dependence of the ratio between the magnitudes of the positron binary to the recoil peak. Such an observation is not effected by the extraction field and should be quite reliable. Here, we find excellent agreement with the experimental observations [5], with the computations and data both displaying values that slowly rise from near unity for $2.4 \mathrm{eV}$ ionized electrons to a factor of 3 for $20 \mathrm{eV}$ ionized electrons. Interestingly, for electron impact, this energy dependence is much shallower, with the ratio only rising to a factor of 1.6 for $20 \mathrm{eV}$ ionized electrons. This we attribute to the opposite post-collision interactions for positron and electron impact.

From these data and the early calculations of Brauner and Briggs [7], one is lead to expect a "classic" binary and recoil peak structure in the FDCS. However, as we turn to $200 \mathrm{eV}$ impact, such predictions fail. During the last few years, Lohmann et al. presented FDCS for the electron- $\operatorname{Ar}(3 p)$ system at impact energies of 200 and $113.5 \mathrm{eV}$ at a fixed projectile scattering angle of $15^{\circ}$ [26-28]. Those FDCS showed a fourlobe pattern and since they were published, they have challenged the state-of-the-art theoretical methods [19,28,29]. No theoretical method has so far provided an ultimate description of the experimental data in the intermediate to low impact energy region which is still subject of active research.

In Fig. 3, we show $200 \mathrm{eV}$ FDCS for positron and electron-impact single ionizations of argon. The electron emission energies under study are similar to those used in the published $500 \mathrm{eV}$ positron data and are 2 and $5 \mathrm{eV}$, while the projectile scattering angles considered are $3.5^{\circ}, 10^{\circ}$, and $15^{\circ}$. In such a comparison, we start with soft collisions with lowmomentum transfer to the argon target $\left(3.5^{\circ}\right)$ and proceed to harder collisions with larger momentum transfer $\left(15^{\circ}\right)$. The classic two-lobe structure is displayed only when the projectile scattering angle (or the momentum transfer) is very small. As the momentum transfer to the target increases, a transition to four-lobe FDCS is realized. Inspection of the partial contributions of the argon $3 p_{0}$ and $3 p_{1}$ orbitals to the FDCS shows that as the momentum transfer increases, the contribution from the $3 p_{0}$ orbital dominates over that of the $3 p_{1}$, leading to a four-lobe pattern in the overall FDCS.

The general dependence of the cross sections on projectile charge can be inferred from several features in the angular distributions. First, we observe for positron impact that the probability for electron emission in the direction of the receding projectile is larger than that corresponding to the electron-impact case. This is due to the post-collision inter- 

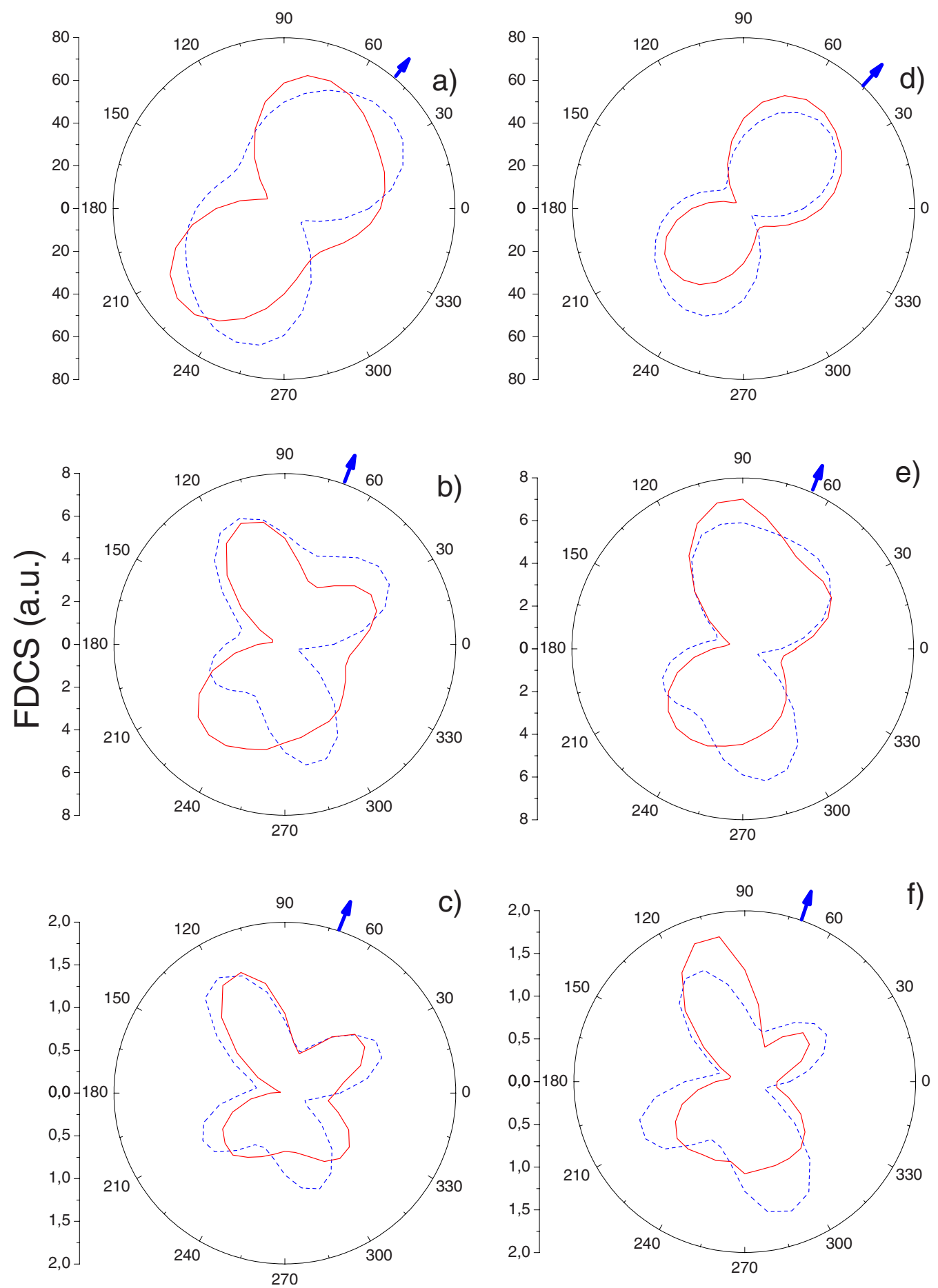

FIG. 3. (Color online) FDCSs for $200 \mathrm{eV}$ positron (solid line) and electron-impact (dashed line) ionizations of the $3 p$ shell of argon. The emitted electron energies are $2 \mathrm{eV}$ (left column) and $5 \mathrm{eV}$ (right column). The projectile scattering angles are $3.5^{\circ}$ (cases a and d), $10^{\circ}$ (cases $\mathrm{b}$ and e), and $15^{\circ}$ (cases $\mathrm{c}$ and $\mathrm{f}$ ) measured clockwise. The small arrows indicate the momentum-transfer direction.

action between the projectile and the ionized electron with the positively charged $e^{+}$accentuating the ionized electron intensity in the forward direction when compared to that of $e^{-}$projectiles. Second, the recoil peak structure is generally more intense for the electron-impact case, showing that the repulsive nature of the projectile-ionized electron interaction results in a stronger momentum exchange with the $\mathrm{Ar}^{+}$ion core. It is clear from Fig. 3 that recalling a simple picture with a single binary and recoil peak is not appropriate when dealing with a complex target such as argon. On the other hand, a four-lobe pattern should not be automatically expected irrespective of the amount of momentum transferred by the projectile. We observe that the two-lobe pattern smoothly changes to a four-lobe pattern as the projectile scattering angle is varied from $3.5^{\circ}$ to $15^{\circ}$. Hence, the use of simple physical pictures for the interpretation of the FDCS 
should be taken with caution since the resulting pattern depends strongly on the momentum transferred by the projectile to the target.

\section{CONCLUSIONS}

To summarize, we have presented a theoretical study of $e^{-}$ and $e^{+}$ionizing collisions from argon at the fully differential level. Our calculations indicate that the FDCS are more complicated than what would be predicted from studies based on radially symmetric targets such as $\mathrm{H}$ or $\mathrm{He}$. The reason we chose the argon target was because it is now a subject of active experimental investigation [5,6,25-28]. Whereas at the impact energy of $500 \mathrm{eV}$ the CDW-EIS predicts a binary peak position close to the momentum-transfer direction, as shown by the recent experimental data by Kheifets et al. [25] for electron impact, we observe clear differences with the recently published positron impact data of de Lucio et al. Although we nicely reproduce the ratio of magnitudes of the positron impact binary to recoil peaks at different emission energies, our binary peaks are located close to the direction in which the momentum-transfer vector is defined in contrast with the data which exhibit binary peaks close to $90^{\circ}$. It may be possible that the convolution of the theoretical FDCS over the reported experimental resolutions would reconcile the agreement with the data. However, such an investigation would exhaust present computing capabilities due to the wide energy and angular acceptances of the data. Further experimental exploration of these geometries involving lowmomentum transfers would be welcome to clarify on this issue. We expect that in the near future, expanded measurements of positron and electron-impact ionizations of argon will provide a step forward in elucidating the collision dynamics for matter-antimatter collisions.

\section{ACKNOWLEDGMENTS}

Work at UNS supported by Contracts No. PGI 24/F038 and No. PICT-2007-00887 of the ANPCyT and through Contract No. PIP 112-200801-02760 of CONICET (Argentina).
[1] H. Ehrhardt, M. Schulz, T. Tekaat, and K. Willmann, Phys. Rev. Lett. 22, 89 (1969).

[2] A. Kover, G. Laricchia, and M. Charlton, J. Phys. B 26, L575 (1993).

[3] Á. Kövér and G. Laricchia, Phys. Rev. Lett. 80, 5309 (1998).

[4] Á. Kövér, K. Paludon, and G. Laricchia, J. Phys. B 34, L219 (2001).

[5] O. G. de Lucio, J. Gavin, and R. D. DuBois, Phys. Rev. Lett. 97, 243201 (2006).

[6] R. D. DuBois, O. G. de Lucio, and J. Gavin, Nucl. Instrum. Methods Phys. Res. B 266, 397 (2008).

[7] M. Brauner and J. S. Briggs, J. Phys. B 26, 2451 (1993).

[8] R. A. Sparrow and R. E. Olson, J. Phys. B 27, 2647 (1994).

[9] J. Fiol, V. D. Rodriguez, and R. O. Barrachina, J. Phys. B 34, 933 (2001).

[10] D. R. Schultz, R. E. Olson, and C. O. Reinhold, J. Phys. B 24, 521 (1991).

[11] L. H. Andersen, P. Hvelplund, H. Knudsen, S. P. Møller, K. Elsener, K. G. Rensfelt, and E. Uggerhøj, Phys. Rev. Lett. 57, 2147 (1986).

[12] D. S. F. Crothers and J. F. McCaan, J. Phys. B 16, 3229 (1983).

[13] P. D. Fainstein, V. H. Ponce, and R. Rivarola, J. Phys. B 24, 3091 (1991).

[14] J. E. Miraglia, Phys. Rev. A 79, 022708 (2009).
[15] M. McCartney and D. S. F. Crothers, J. Phys. B 26, 4561 (1993).

[16] L. Gulyas, P. D. Fainstein, and A. Salin, J. Phys. B 28, 245 (1995).

[17] L. Gulyás and T. Kirchner, Phys. Rev. A 70, 022704 (2004).

[18] S. Jones and D. H. Madison, Phys. Rev. Lett. 81, 2886 (1998)

[19] S. Otranto, Phys. Rev. A 79, 012705 (2009).

[20] M. Gell-Mann and M. L. Goldberger, Phys. Rev. 91, 398 (1953).

[21] F. A. Gianturco and S. Scialla, J. Phys. B 20, 3171 (1987).

[22] E. Clementi and C. Roetti, At. Data Nucl. Data Tables 14, 177 (1974).

[23] A. W. Malcherek and J. S. Briggs, J. Phys. B 30, 4419 (1997).

[24] J. R. Götz, M. Walters, and J. S. Briggs, J. Phys. B 36, L77 (2003).

[25] A. S. Kheifets, A. Naja, E. M. Staicu Casagrande, and A. Lahmam-Bennani, J. Phys. B 41, 145201 (2008).

[26] M. A. Haynes and B. Lohmann, Phys. Rev. A 64, 044701 (2001).

[27] M. Stevenson, G. J. Leighton, A. Crowe, O. K. Vorov, and D. H. Madison, J. Phys. B 38, 433 (2005).

[28] M. A. Stevenson and B. Lohmann, Phys. Rev. A 77, 032708(R) (2008).

[29] A. Prideaux, D. H. Madison, and K. Bartschat, Phys. Rev. A 72, 032702 (2005). 